\title{
Effect of aging on H-reflex response to fatigue
}

\author{
Andrew Philip Lavender ${ }^{1}$, Sadik Balkozak ${ }^{2 \uparrow, ~ M u s t a f a ~ G o ̈ r k e m ~ O ̈ z y u r t ~}{ }^{3 \uparrow, ~ B e t i l a y ~ T o p k a r a ~}{ }^{3}$, İlhan Karacan ${ }^{4}$, İdil \\ Bilici $^{5}$, Anne-Marie Hill ${ }^{1}$, Kemal Sitki Türker ${ }^{3 *}$. \\ IEqual Contribution
}

1. School of Physiotherapy and Exercise Science, Curtin University, Perth, Australia.

2. Faculty of Medicine, Dokuz Eylul University, Izmir, Turkey.

3. School of Medicine, Koç University, Istanbul, Turkey.

4. Department of Physical Medicine, Istanbul Training and Research Hospital, Istanbul, Turkey.

5. Faculty of Medicine, Ege University, Izmir, Turkey.

\author{
Corresponding Author*: \\ Kemal S. Türker, BDS, PhD \\ Professor of Physiology \\ Koç University School of Medicine \\ Istanbul, Turkey 34450 \\ E-mail: kturker@ku.edu.tr
}

Tel: +90 2123381174

Fax: +90 2123381165 


\section{ABSTRACT}

Injury as a result of tripping is relatively common among older people. The risk of falling increases with fatigue and of importance is the ability to dorsiflex the foot through timely activation of the tibialis anterior (TA) muscle to ensure the foot clears the ground, or an obstacle, during the swing phase of walking. We, therefore, questioned whether the muscle spindle input to the motoneurons alter with ongoing fatigue in elderly people. We electrically stimulated the common peroneal nerve to assess the TA primary afferent efficacy using H-reflex before, immediately following and after a fatiguing maximal isometric contraction. M-response was kept unchanged throughout the experiment to ensure a similar stimulus intensity was delivered across time points. H-reflex increased significantly while the TA muscle was in a state of fatigue for the younger participants but tended to decrease with increasing age. The main contributor to the tonicity of TA muscle, i.e., excitatory synapses of spindle primary endings of motoneurons that innervate TA muscle, tend to lose their efficacy during fatigue in the older individuals but increased efficiency in the majority of the younger people. Since TA muscle is the main dorsiflexor of the foot and it needs to be active during the swing phase of stepping to prevent tripping, older individuals become more susceptible to falling when their muscles are fatigued. This finding may help improve devices/treatments to overcome the problem of tripping among older individuals.

Keywords: Fatigue, aging, H-reflex, tibialis anterior, human

\section{ABBREVIATIONS}

\begin{tabular}{|l|r|}
\hline BMI & Body-mass index \\
\hline H-reflex & Hoffmann reflex \\
\hline Mmax & Maximum direct motor response \\
\hline M-response & Direct motor response \\
\hline MVC & Maximum voluntary contraction \\
\hline ROM & Range of motion \\
\hline SD & Standard deviation \\
\hline SEMG & Surface electromyography \\
\hline TA & Tibialis anterior \\
\hline
\end{tabular}




\section{INTRODUCTION}

Incidences of older individuals falling presents a major societal health problem as it can lead to serious injury (Bergen et al. 2016). A fall resulting in severe injury such as hip fracture and traumatic brain injury (Stel et al. 2004) increases the likelihood of further complications leading to morbidity, hospitalization and institutionalization among community dwelling elders (Elliott and Leland 2018). Falls among older people has become such a serious global socioeconomic issue that in 2007 the World Health Organization (WHO) recommended a proactive systematic approach to developing policies and strategies for falls prevention tailored to target older populations (WHO). There are several factors that may contribute to making a person more likely to experience a fall including poor vision, reduced mobility skills, side effects of medication, improper footwear and environmental hazards (Bloch et al. 2010; Deandrea et al. 2010). Older individuals experience age-related changes in visual, vestibular, proprioceptive and neuromuscular systems which increase their risk of falling (Kerrigan et al. 1998; Shaffer and Harrison 2007; Stelmach et al. 1990; Tang and Woollacott 1998).

A study of activation of leg muscles in young and older subjects when tripped showed that several muscles were late to activate in the older group. The likelihood of tripping increases greatly with fatigue due to adjustments to gait such as increased step width, longer double support and greater minimum foot clearance to compensate for a reduction in balance ability regardless of age (Nagano et al. 2014). For older individuals, having reduced muscle mass, strength (Landi et al. 2018) and aerobic capacity (Kirkendall and Garrett 1998), fatigue is important since it can affect balance and increase the likelihood of stumbling. After a trip occurs, slower reaction times in older individuals means that they are less able to regain balance after it is lost, resulting in a fall (Morrison et al. 2016). A systematic review presents studies in gait adjustments for stepping over an object in young and older people. The older volunteers tend to overcompensate by increasing hip and knee flexion, hip abduction and ankle dorsiflexion to ensure hazard clearance. Yet with a time constraint, older subjects are more likely to trip (Galna et al. 2009).

Postural sway during standing is greater in older than young subjects (Tavares et al. 2017) and cortical proprioceptive processing is impaired with ageing (Piitulainen et al. 2018). So, a decline in balance may be partially due to reduced proprioception and ability of the cortex to process sensory information (Piitulainen et al. 2018). These aging-related functional declines result in an increased likelihood of falling when fatigued.

During walking, timing and amplitude of muscle activation is important for optimal function. During stair climbing older subjects have been shown to have less dorsiflexion of the ankle than younger volunteers (Benedetti et al. 2007). During walking while apprehensive, induced by a cognitive dual task, muscle activation level and co-contraction of the triceps surae and tibialis anterior (TA) muscles increases more in older subjects than 
young (Hallal et al. 2013). This co-contraction causes reduced efficiency of ankle dorsiflexion in the swing phase with older people expending $21 \%$ more energy than younger subjects due to co-contraction of TA and triceps surae during normal walking increasing the chance for the toes to contact the ground during the swing phase (Mian et al. 2006) .

Muscle spindles help coordinate muscle activation during standing (Aniss et al. 1990), stepping (Cronin et al. 2009) and bouncing gaits such as running and hopping (Ellaway et al. 2015; Geyer et al. 2003). Early rat (Andrew et al. 1978) and cat (Bennett et al. 1996; Taylor et al. 2006) studies show that gamma motor efferents are predominantly responsible for activation of soleus extrafusal fibers in quiet standing. The gamma system and muscle spindles are vital for maintaining posture during standing, walking and running (Takakusaki 2017). In fact, one of the major functions of the muscle spindles is to stabilize the body during actions such as walking (Taylor et al. 2006; Zehr and Stein 1999). This is achieved by the gamma system which induces activation of both the agonist and antagonist (Taylor et al. 2006) of, for example, the ankle joint in order for that particular leg to be moved with the rigid foot following it without dangling during stepping.

In this study we attempt to discover whether effectiveness of spindle afferent input into motoneurons that innervate the primary muscles that extend the toes and dorsiflex the foot (Nath and Somasundaram 2017) changes during fatigue and whether the changes are related to age. Our principle hypothesis is that the spindle input will be different between young and old and this is exaggerated during fatigue.

\section{METHODS}

Experiments were performed in the neurophysiology laboratory of Koç University. All participants gave written informed consent to the experimental procedures, which were in accordance with the Declaration of Helsinki and were approved by the University's Human Ethics Committee (2017.124.IRB2.038). Forty-nine volunteers aged between 18 and 80 years were assessed in this study. Nine of the subjects were female (average age; $35.9 \pm 14.1 \mathrm{yrs}$ $[$ mean $\pm \mathrm{SD}]$ and body mass index $[\mathrm{BMI}] ; 22.9 \pm 2.0 \mathrm{~kg} / \mathrm{m}^{2}$ ) and forty were male (average age; $48.2 \pm 19.9 \mathrm{yrs}$, BMI; $\left.25.3 \pm 3.1 \mathrm{~kg} / \mathrm{m}^{2}\right)$. The age distribution for subjects was 18-29 ( $\left.=13\right), 30-39(\mathrm{~N}=7), 40-49(\mathrm{~N}=7), 50-59$ $(\mathrm{N}=7), 60-69(\mathrm{~N}=7)$ and 70-80 $(\mathrm{N}=7)$ yrs. To standardize the body shape, we only included subjects with a BMI value between 20 to $31 \mathrm{~kg} / \mathrm{m}^{2}$. All were selected from population of Koç University and Sariyer district and were healthy as well as free from any neuromuscular disorders.

\subsection{Setup}

The Spike2 7.20 software package (Cambridge Electronic Designs, Cambridge, UK) was used with 
CED 1902 Quad System MKIII amplifier and CED 3601 Power 1401 MKII digital to analogue converter (DAC) to record surface electromyography (SEMG) and force data. A constant current stimulator (model DS7A, Digitimer Ltd, Hertfordshire, UK) was used to deliver both twitch interpolation and H-reflex stimuli. A force plate attached to a linear strain gauge (Model LC1205-K020, A \& D Co. Ltd., Tokyo, Japan: linear to 196 N) was used to quantify the amount of twitch produced with electrical stimulation (Fig 1).

\subsection{Surface Electromyography}

SEMG was recorded with self-adhesive bipolar $\mathrm{Ag} / \mathrm{AgCl}$ electrodes placed over the belly of the TA muscle of the right leg in a longitudinal alignment with $4 \mathrm{~cm}$ interelectrode distance (Tucker and Türker 2005) (Fig 1a). Skin resistance was tested to ensure the connection was optimal (less than $10 \mathrm{k} \Omega$ ). SEMG activity was filtered at $20-10,000 \mathrm{~Hz}$ band pass filter and recorded with 20,000 $\mathrm{Hz}$ sampling rate. The skin over the TA muscle of each subject was prepared by first shaving the area then rubbing it gently with a very fine grade of sandpaper and cleaning with an alcohol swab. Gel was applied to the recording site and after allowing the gel to soak into the skin for a few moments it was rubbed off with a clean tissue. The same preparations were done on the lateral malleolus on which ground electrode for recording was placed.

\subsection{Force Recording}

The right foot was tightly strapped to the force plate and secured with Velcro tape. Tight fixation of the foot was ensured to prevent loss of force and to make sure that the dorsiflexion was isometric (Fig 1b). Force signals were amplified 1,000 times, filtered with DC-100 Hz and sampled at 2,000 Hz.

\subsection{Experimental Procedure}

Subjects were asked to lie down in a prone position on a plinth and their right foot was secured to a force plate located at the foot of the plinth. After placement of the SEMG electrodes, exact position of the stimulating electrodes was determined as follows; i) head of fibula was located, ii) custom made anode and cathode with $2 \mathrm{~cm}$ interelectrode distance was placed just distal to the head of fibula, iii) an evoked M-response without voluntary contraction and H-reflex with slight voluntary contraction using $1 \mathrm{~ms}$ width square pulses was found and that location was determined as the hotspot for the stimulation in the experiment.

The next step was to find the maximum M-response (Mmax) that was achieved by increasing the stimulus intensity up to a point where no further increment in M-response was observed. This stimulus intensity was noted and 1.5x this level was used for twitch interpolation. Maximum voluntary contraction (MVC) level using SEMG of TA muscle was then determined by asking the subject to perform maximal dorsiflexion 3 times with verbal encouragement from the researchers. The maximum of three attempts, each lasting for $3 \mathrm{~s}$ with a resting 
period of $30 \mathrm{~s}$ in between, was selected as the MVC. After that, 10\% of MVC was determined and displayed to subjects on a monitor and they were asked to keep their contraction steady at the target level to facilitate generation of H-reflex. Determination of the EMG levels, including the visual feedback provided to subjects, were done using rectified and smoothed (by $0.2 \mathrm{~s}$ ) EMG. Before the experiment subjects were trained several minutes to familiarize with the feedback monitor and the level of contraction to achieve their own 10\% of MVC. In addition, one of the researchers provided verbal feedback to make sure that the subjects contract the muscle at $10 \%$ of MVC during Hreflex testing.

After a rest period of one minute, the experimental protocol was initiated. Subjects were asked to perform dorsiflexion at $10 \%$ of MVC and to hold that level during the stimulation period and then, to obtain $\mathrm{H}$ reflex, 30 square pulses with $1 \mathrm{~ms}$ width were delivered with an interstimulus interval of 1-2 seconds as the first prefatigue assessment, followed by a 2-minute resting period. Randomly delivered stimuli at this rate has been reported not to cause post activation depression so long as the muscle is contracting actively (Burke et al. 1989). H-reflex was obtained by applying bipolar electrical stimulation to the common peroneal nerve just below the head of fibula (Fig 1a). Stimulus intensity was set to generate an $\mathrm{H}$-reflex together with an average M-response of about $15-20 \%$ of Mmax.

Thirty seconds before the next step, several stimuli were delivered to make sure that the cathode position did not shift by measuring peak-to-peak M-response in real-time. If the M-response amplitude was reduced due to slight change of cathode position, the stimulation intensity was increased slightly to keep the M-response amplitude similar with the previous protocol, or if the M-response was increased then stimulus intensity was reduced to keep the level same.

Next, 30 more stimuli were delivered at the same intensity in the $2^{\text {nd }}$ stimulation block before the fatiguing maximal isometric contraction. As soon as the last stimulus was delivered, the subject was asked to contract TA muscle as much as he/she can with a continuous verbal encouragement from the researchers. During this fatiguing period, a pair of $100 \mathrm{~Hz}$ stimuli with $1.5 \mathrm{x}$ supramaximal threshold intensity were delivered (Fig 1c) every 10-20 s (Herbert and Gandevia 1999). The real-time twitch production was monitored, and fatigue was determined with an objective measurement: i) the maximum persistent force production was reduced to below $50 \%$ of MVC and ii) twitch produced by supramaximal stimuli could not increase the force level over $50 \%$ of MVC. Hence, the subject was regarded as fatigued if their force generation capacity was reduced to below $50 \%$ of their own maximum with and without electrical stimulation. At this time subjects were immediately asked to keep their contraction level at $10 \% \mathrm{MVC}$ and 30 stimuli at the same settings were delivered (fatigue assessment set). This period was followed by 2 sets of 30 stimuli post-fatigue with 2 minutes of rest in between. 


\subsection{Analysis}

The waveform average of trains of stimuli for each set was done using Spike2 software. The peak-topeak value of average M-response and H-reflex, located between the blue markers for $\mathrm{M}$ and red markers for $\mathrm{H}$, were calculated as shown in Fig 2. Moreover, a sample recording of determination of the fatigue has been shown in Fig 2c where the force generation no longer goes above $50 \%$ of their own MVC even with the electrical stimulation. The amplitudes in all 5 trials ( $2 x$ pre-fatigue, fatigue and $2 x$ post-fatigue) were calculated. The measured responses were normalized to each subjects' own Mmax to provide a percent response. Then, the two pre and two post values were averaged to provide one pre-fatigue value and one post-fatigue value for H-reflex. This was also done for Mresponse to see if M-response remained similar between sets. The percent reflex change between pre and fatigue was calculated by subtracting the pre-fatigue H-reflex value from fatigue H-reflex and dividing by pre-fatigue H-reflex amplitude (Pre-fatigue vs Fatigue), and this was done in the same way for Pre-fatigue vs Post-fatigue. The percentage change of H-reflex for each subject was fitted to a linear regression line to correlate the reflex amplitude changes with age. For the M-response, Shapiro-Wilk test was used to test their distribution and Wilcoxon matchedpairs signed rank test was used to investigate their difference. The level of significance was determined as $\mathrm{p}<0.05$. GraphPad Prism 8 was used for statistical analysis.

\section{RESULTS}

Linear regression showed that change in the H-reflex amplitudes during the fatigue period was significantly higher in the younger people but decreased with increasing age $\left(R^{2}=0.3735, p<0.0001\right)$ compared to pre-fatigue condition, illustrated in Fig 3. The level of M-response between pre-fatigue and fatigue period was found to be insignificant ( $\mathrm{p}=0.1876$ ), revealing similar effective stimulus intensity were delivered in each protocol.

After the fatigue period, recovery of the H-reflex was not correlated with the age and showed no significant change $\left(\mathrm{R}^{2}=0.0069, \mathrm{p}=0.5691\right)$ which was also the case for the M-response $(\mathrm{p}=0.2063)$ (Fig 4).

\section{DISCUSSION}

This study has led to two original findings: Firstly, that synaptic efficacy of muscle spindle primary endings in TA muscle changes significantly during fatigue. Secondly, that during fatigue, spindle efficacy increases in the young while it decreases in the old.

Falls in older people often occur as a result of tripping (Hundza et al. 2018). This is likely related to 
physiological differences between young and older people. Aging results in several changes in the physiology of joints, including a reduced water content of cartilage, synovial fluid volume and proteoglycans which leads to increased stiffness (Hamerman 1998). This increased stiffness reduces range of motion (ROM) of the ankle in older people leading to compromised functional ability.

The TA muscle is primarily responsible for dorsiflexion of the foot and its timely activation during the walking gait cycle is important for achieving minimal foot clearance during the swing phase of walking which is fundamental for avoidance of tripping (Nagano et al. 2015). Dorsiflexion ROM of the first metatarsophalangeal joint can be reduced by as much as $32 \%$ in older people compared with their younger counterparts due to joint stiffness (Scott et al. 2007). This reduced range of motion combined with loss of strength through sarcopenia likely contributes to an increased risk of tripping among older individuals due to insufficient activation of TA when the muscle is fatigued. The ability for people to adapt to changes in terrain due to this reduction in ankle dorsiflex is strongly correlated with poor balance and function among older people (Menz et al. 2005; Spink et al. 2011). In fact, one study has shown that the age-related decrease in the ability to dorsiflex the ankle is a risk factor for falling (Menz et al. 2006).

One of the most common causes of falling among older people is tripping over an object (Lord et al. 1993). This can result in severe injury and may be related to many factors including ill-fitting footwear, poor vision and balance (Deandrea et al. 2010). Fatigue is also a major contributor to falls among older individuals as strength and force production capability are reduced while muscles are in a state of fatigue (Hatton et al. 2013). During fatigue, voluntary movements and the response to a perturbation are slowed which can impair function so that regaining balance when it is lost is not possible and a fall then occurs. Older individuals compensate for reduced function while fatigued by altering their gait to wider steps, increasing variability in step length and altering trunk acceleration (Helbostad et al. 2007). Rather than improving their ability to keep their center of mass within the base of support, these changes may, in fact, reduce their stability while walking in a fatigued state making a fall more likely. Conversely, one study showed that fatigue of the knee extensors resulted in faster walking, longer stride length and less variability in stride length among older volunteers (Granacher et al. 2010). The authors suggest that the tendency for the older volunteers to increase their speed was a strategic decision to try to complete the task more quickly to relieve the discomfort associated with fatigue. Clearly, further investigation on this topic is warranted.

The results from the present study align with previous research which suggests that immediately after a fatiguing contraction, while the subjects perform a submaximal isometric contraction an increase in amplitude of the H-reflex relative to M-response becomes evident for some muscles (Stutzig and Siebert 2017). This was the case for TA muscle in the younger subjects in the present study. Interestingly, some studies investigating plasticity of neural 
pathways following a fatiguing contraction stimulated the nerve while the muscle was at rest and found the opposite effect, that H-reflex was reduced immediately after a fatiguing contraction relative to the M-response (Duchateau et al. 2002; Walton et al. 2002). The consensus from the authors of the above-mentioned studies was that the underlying mechanisms for the differences in findings are likely multi-faceted since there were differences in the protocols of these studies other than the activation of the muscle under investigation (Stutzig and Siebert 2017). The fatiguing protocols used in previous studies varied with fatigue being induced with voluntary isometric contractions, voluntary dynamic contractions or neuromuscular electrical stimulation (Stutzig and Siebert 2017). In this study we have attempted to use a standardized approach to the fatiguing contraction. Volunteers in this study maintained a maximal isometric contraction of TA for as long as they could while the investigators continued to give verbal encouragement. The twitch interpolation method was used to ensure 50\% MVC fatigue was induced.

The previously mentioned study by Stutzig and Siebert (2017) also employed the twitch interpolation method to determine the level of fatigue during a muscle contraction which was maintained using neuromuscular electrical stimulation at $80 \mathrm{~Hz}$ with $5 \mathrm{~s}$ on, 20s off between trains. A superimposed doublet with interstimulus interval of $10 \mathrm{~ms}$ was used in the same way as the present investigation to assess the level of fatigue while volunteers were maintaining an isometric contraction of $20 \%$ MVC. This is a valid method for ensuring peripheral fatigue has been achieved as the twitch does not rely on central control (Herbert and Gandevia 1999). The volunteers in our study achieved a fatigue of $50 \% \mathrm{MVC}$ force with the twitch interpolation although the level of activation was $10 \%$ of the rested MVC measured using SEMG. Using this method, we can be sure that the muscle is in a state of fatigue however the fatigue-test must be done immediately after the fatiguing isometric contraction as recovery can be quick as shown in the current post-test data.

During each experiment the M-response was monitored to make sure it remained at the same level throughout the testing session. This ensures that the effective stimulus was kept stable throughout the experiment. Therefore, any change in the H-reflex response would represent a genuine alteration in the efficacy of the spindle primary afferent synapse on the motoneuron pool that innervates the tibialis anterior muscle.

Studies of older age groups have shown that ageing is associated with functional decrements in balance, force production capacity, reaction time and proprioception as discussed by Shaffer and Harrison (2007). Also, Hatton et al. (2013) showed that fatigue of the lower limbs in older volunteers impairs movement control and accuracy of foot placement before and after negotiating low-level obstacles. When these are compromised by fatigue, tripping becomes more likely.

Fatigue is described as a failure to maintain power output. Peripheral fatigue occurs as a result of failure of biological functions caused by high intensity repeated activity and may involve the motoneuron, neuromuscular 
junction, sarcolemma membrane, excitation-contraction coupling, accumulation of metabolites or depletion of fuel sources (Kirkendall 1990). Central fatigue describes the failure of muscles to produce force as the result of reduced activation of the motor cortex decreasing recruitment of the motoneuron pool at the spinal level (Gandevia 2001). It is the combination of these that results in the eventual failure of the participant to continue the task. A recent study that compared aerobically induced fatigue of large and small muscles between young and older volunteers found that, although the younger group performed significantly more work than the older group, their peripheral fatigue level was less than that of the older group while their central fatigue level was greater (Weavil et al. 2018). An important variable in the study by Weavil et al. (2018) is the physical activity level of participants and the authors were careful to match physical activity between the groups. Since the aerobic fitness level of the individuals in each of the groups is similar, it is interesting that age was a determining factor for both central and peripheral fatigue following both knee extension and cycling exercise. The authors explained that this may be due to neural feedback from group III/IV muscle afferents and neural feedforward.

In the present study, the difference between younger and relatively older adults is that, while the change in H-reflex among the younger subjects aligned with previous research, that is it increased during a submaximal contraction while fatigued (Stutzig and Siebert 2017), the changes in H-reflex for the older group were the opposite. H-reflex amplitude decreased with increasing age.

\subsection{Conclusions}

This study is in line with previous research that shows fatigue affects H-reflex of a lower leg muscle. This investigation shows that there is a difference in response of the H-reflex to a voluntary fatiguing isometric contraction of the TA muscle between young and relatively older adults. The TA muscle was chosen as the muscle of interest for this study as it plays a vital role in dorsiflexion of the foot which can be compromised if the TA is fatigued. This becomes more important when an individual has poor balance as is the case for many older individuals. Since the timely activation of the TA muscle during walking is vital for avoidance of a fall, the current study suggests that exercises to maintain strength and fatigue resistance of this muscle is relevant for older individuals.

\subsection{Limitations of the Study}

We used SEMG of the TA muscle for determining its MVC. This is due to the fact that although SEMG represents the activation of motor units in a given muscle, dorsiflexion force is induced by a combination of muscles including the antagonists (Scutter and Turker 1998). Therefore, SEMG of one of the contributing muscles, i.e. TA, was our method of choice for determining the MVC of the TA muscle. However, for the fatiguing contraction and 
twitch interpolation, we used the dorsiflexion force. This is due to the fact that published literature describes muscular fatigue as "the reduction in force generating capacity" and we were also stimulating the muscles' nerve for twitch generation. We suggest that, although this may look controversial, SEMG is the optimal way to activate the motor units in a muscle of interest at a given level of drive, i.e. 10\% MVC. We also note that $10 \% \mathrm{MVC}$ before fatigue and immediately after fatigue may represent activation of a different population of motor units. This problem would have been compounded if force was used for feedback, as the contribution of TA to $10 \% \mathrm{MVC}$ force would vary since there are abundant ways to generate $10 \% \mathrm{MVC}$ force in a limb where several muscles contribute to its force production. In addition, number of female participants was lower compared to male.

\section{ACKNOWLEDGMENTS}

We would like to thank participants who attended this study. Also, we acknowledge the role of Koç University School of Medicine for supporting this study. We also thank the Scientific and Technological Research Council of Turkey (TÜBİTAK) for funding Mustafa Görkem Özyurt under 2211-A programme. This research did not receive any specific grant from funding agencies in the public, commercial, or not-for-profit sectors.

\section{AUTHOR CONTRUBITIONS}

APL, MGO, BT, IK, AMH and KST conceived the study design. APL, SB, MGO, BT, and IB performed the experiments. SB, MGO, BT, and IB analyzed the results. APL, MGO, IK and KST wrote the first draft of the manuscript and all the authors approved the final version of the manuscript.

\section{DISCLOSURES}

The authors declare that they have no conflict of interest.

\section{REFERENCES}

1. Andrew BL, Leslie GC, Part NJ (1978) Some observations on the efferent innervation of rat soleus muscle spindles. Exp Brain Res 31(3):433-443.

2. Aniss AM, Diener HC, Hore J, Gandevia SC, Burke D (1990) Behavior of human muscle receptors when reliant on proprioceptive feedback during standing. J Neurophysiol 64(2):661-670.

3. Benedetti MG, Berti L, Maselli S, Mariani G, Giannini S (2007) How do the elderly negotiate a step? A biomechanical assessment. Clin Biomech 22(5):567-573.

4. Bennett DJ, De Serres SJ, Stein RB (1996) Regulation of soleus muscle spindle sensitivity in decerebrate 
and spinal cats during postural and locomotor activities. J Physiol 495(3):835-850.

5. Bergen G, Stevens MR, Burns ER (2016) Falls and fall injuries among adults aged $>/=65$ years - United States, 2014. MMWR Morb Mortal Wkly Rep 65(37):993-998.

6. Bloch F, Thibaud M, Dugué B, Brèque C, Rigaud A, Kemoun G (2010) Episodes of falling among elderly people: a systematic review and meta-analysis of social and demographic pre-disposing characteristics. Clinics 65:895-903.

7. Burke D, Adams RW, Skuse NF (1989) The effects of voluntary contraction on the H reflex of human limb muscles. Brain 112 (Pt 2): 417-433.

8. Cronin NJ, Ishikawa M, Grey MJ, Af Klint R, Komi PV, Avela J, Sinkjaer T, Voigt M (2009) Mechanical and neural stretch responses of the human soleus muscle at different walking speeds. J Physiol 587(13):3375-3382.

9. Deandrea S, Lucenteforte E, Bravi F, Foschi R, La Vecchia C, Negri E (2010) Review Article: Risk Factors for Falls in Community-dwelling Older People: "A Systematic Review and Meta-analysis". Epidemiology 21(5):658-668.

10. Duchateau J, Balestra C, Carpentier A, Hainaut K (2002) Reflex regulation during sustained and intermittent submaximal contractions in humans. J Physiol 541(3):959-967.

11. Ellaway PH, Taylor A, Durbaba R (2015) Muscle spindle and fusimotor activity in locomotion. J Anat 227(2):157-166.

12. Elliott S, Leland NE (2018) Occupational Therapy Fall Prevention Interventions for Community-Dwelling Older Adults: A Systematic Review. Am J Occup Ther 72(4):7204190040p7204190041 $7204190040 p 7204190011$.

13. Galna B, Peters A, Murphy AT, Morris ME (2009) Obstacle crossing deficits in older adults: A systematic review. Gait Posture 30(3):270-275.

14. Gandevia SC (2001) Spinal and Supraspinal Factors in Human Muscle Fatigue. Physiol Rev 81(4):17251789.

15. Geyer H, Seyfarth A, Blickhan R (2003) Positive force feedback in bouncing gaits? P Roy Soc Lond B Bio 270(1529):2173.

16. Granacher U, Wolf I, Wehrle A, Bridenbaugh S, Kressig RW (2010) Effects of muscle fatigue on gait characteristics under single and dual-task conditions in young and older adults. J Neuroeng Rehabil $7(1): 56$.

17. Hallal CZ, Marques NR, Spinoso DH, Vieira ER, Gonçalves M (2013) Electromyographic patterns of lower limb muscles during apprehensive gait in younger and older female adults. J Electromyogr Kinesiol 
23(5):1145-1149.

18. Hamerman D (1998) Biology of the aging joint. Clin Geriatr Med 14(3):417-433.

19. Hatton AL, Menant JC, Lord SR, Lo JCM, Sturnieks DL (2013) The effect of lower limb muscle fatigue on obstacle negotiation during walking in older adults. Gait Posture 37(4):506-510.

20. Helbostad JL, Leirfall S, Moe-Nilssen R, Sletvold O (2007) Physical Fatigue Affects Gait Characteristics in Older Persons. J Gerontol A-Biol 62(9):1010-1015.

21. Herbert RD, Gandevia SC (1999) Twitch interpolation in human muscles: mechanisms and implications for measurement of voluntary activation. J Neurophysiol 82(5):2271-2283.

22. Hundza SR, Gaur A, Brodie R, Commandeur D, Klimstra MD (2018) Age-related erosion of obstacle avoidance reflexes evoked with electrical stimulation of tibial nerve during walking. J Neurophysiol 119(4):1528-1537.

23. Kerrigan DC, Todd MK, Della Croce U, Lipsitz LA, Collins JJ (1998) Biomechanical gait alterations independent of speed in the healthy elderly: Evidence for specific limiting impairments. Arch Phys Med Rehab 79(3):317-322.

24. Kirkendall DT (1990) Mechanisms of peripheral fatigue. Med Sci Sport Exer 22(4):444-449.

25. Kirkendall DT, Garrett WE (1998) The Effects of Aging and Training on Skeletal Muscle. Am J Sport Med 26(4):598-602.

26. Landi F, Calvani R, Cesari M, Tosato M, Martone AM, Ortolani E, Savera G, Salini S, Sisto A, Picca A, Marzetti E (2018) Sarcopenia: An Overview on Current Definitions, Diagnosis and Treatment. Curr Protein Pept Sci 19(7):633-638.

27. Lord SR, Ward JA, Williams P, Anstey KJ (1993) An epidemiological study of falls in older communitydwelling women: the Randwick falls and fractures study. Aust J Public Health 17(3):240-245.

28. Menz HB, Morris ME, Lord SR (2005) Foot and Ankle Characteristics Associated With Impaired Balance and Functional Ability in Older People. The Journals of Gerontology: Series A 60(12):1546-1552.

29. Menz HB, Morris ME, Lord SR (2006) Foot and Ankle Risk Factors for Falls in Older People: A Prospective Study. J Gerontol A-Biol 61(8):866-870.

30. Mian OS, Thom JM, Ardigò LP, Narici MV, Minetti AE (2006) Metabolic cost, mechanical work, and efficiency during walking in young and older men. Acta Physiol 186(2):127-139.

31. Morrison S, Colberg SR, Parson HK, Neumann S, Handel R, Vinik EJ, Paulson J, Vinik AI (2016) WalkingInduced Fatigue Leads to Increased Falls Risk in Older Adults. J Am Med Dir Assoc 17(5):402-409.

32. Nagano H, James L, Sparrow WA, Begg RK (2014) Effects of walking-induced fatigue on gait function and tripping risks in older adults. J Neuroeng Rehabil 11(1):155. 
33. Nagano H, Sparrow WA, Begg RK (2015) Can toe-ground footwear margin alter swing-foot ground clearance? Gait Posture 42(2):214-217.

34. Nath Rahul K, Somasundaram C (2017) Gait Improvements After Peroneal or Tibial Nerve Transfer in Patients with Foot Drop: A Retrospective Study. Eplasty 17:e31.

35. Piitulainen H, Seipajarvi S, Avela J, Parviainen T, Walker S (2018) Cortical Proprioceptive Processing Is Altered by Aging. Front Aging Neurosci 10:147.

36. Scott G, Menz HB, Newcombe L (2007) Age-related differences in foot structure and function. Gait Posture 26(1):68-75.

37. Scutter SD, Turker KS (1998) Recruitment stability in masseter motor units during isometric voluntary contractions. Muscle Nerve 21(10):1290-1298.

38. Shaffer SW, Harrison AL (2007) Aging of the Somatosensory System: A Translational Perspective. Phys Ther 87(2):193-207.

39. Spink MJ, Fotoohabadi MR, Wee E, Hill KD, Lord SR, Menz HB (2011) Foot and Ankle Strength, Range of Motion, Posture, and Deformity Are Associated With Balance and Functional Ability in Older Adults. Arch Phys Med Rehabil 92(1):68-75.

40. Stelmach GE, Zelaznik HN, Lowe D (1990) The influence of aging and attentional demands on recovery from postural instability. Aging Clin Exp Res 2(2):155-161.

41. Stutzig N, Siebert T (2017) Assessment of the H-reflex at two contraction levels before and after fatigue. Scand J Med Sci Spor 27(4):399-407.

42. Tang P-F, Woollacott MH (1998) Inefficient Postural Responses to Unexpected Slips During Walking in Older Adults. J Gerontol A-Biol 53A(6):M471-M480.

43. Tavares JT, Biasotto-Gonzalez DA, Boa Sorte Silva NC, Suzuki FS, Lucareli PRG, Politti F (2017) AgeRelated Changes in Postural Control in Physically Inactive Older Women. J Geriatr Phys Ther 42(3):E81E86.

44. Taylor A, Durbaba R, Ellaway PH, Rawlinson S (2006) Static and dynamic $\gamma$-motor output to ankle flexor muscles during locomotion in the decerebrate cat. J Physiol 571(3):711-723.

45. Tucker KJ, Türker KS (2005) A new method to estimate signal cancellation in the human maximal Mwave. J Neurosci Methods 149(1):31-41.

46. Walton DM, Kuchinad RA, Ivanova TD, Garland JS (2002) Reflex inhibition during muscle fatigue in endurance-trained and sedentary individuals. Eur J Appl Physiol 87(4):462-468.

47. Weavil JC, Hureau TJ, Thurston TS, Sidhu SK, Garten RS, Nelson AD, McNeil CJ, Richardson RS, Amann M, 2018. The Impact of Age on the Development of Fatigue during Large and Small Muscle Mass 
Exercise, Am J Physiol-Reg I, July 11, 2018 ed., pp. R741-R750.

48. WHO, WHO global report on falls prevention in older age https://extranet.who.int/agefriendlyworld/wpcontent/uploads/2014/06/WHo-Global-report-on-falls-prevention-in-older-age.pdf. (Accessed 10 Aug 2018).

49. Zehr EP, Stein RB (1999) What functions do reflexes serve during human locomotion? Prog Neurobiol 58(2):185-205.

\section{Figure Legends}

Fig 1 The experimental setup used in the study. A) The location of SEMG electrodes was on the belly of TA muscle and the stimulating electrode slightly distal of the head of the fibula. B) The position of the subject and placement of the leg against the force transducer. $C)$ The experimental procedure for pre-fatigue $(1,2)$, ongoing fatigue $(F)$ and post-fatigue $(3,4)$ sets

Fig 2 A sample recording of the smoothed SEMG, raw SEMG and force. A) Rectified-smoothed (by 0.2 s) SEMG represents the 10\% MVC background activity which is similar during each of pre-fatigue, fatigue and post-fatigue protocol respectively from left to right. B) Each figure shows an average response to primary afferent stimulation. C) The larger box represents the fatigue development over time in force recording and the inserted smaller box illustrates a magnified view of a twitch produced by paired stimuli. The letter $M$ stands for $M$-response and $H$ for $H$ reflex. The “*” indicates the stimulus artifact. Blue double arrows show the paired supramaximal stimuli. Only one of the pre and post fatigue protocol is illustrated for representation purposes, note that there were 2 pre-fatigue and post-fatigue protocols for each subject

Fig 3 The percent change of H-reflex between pre-fatigue and fatigue as well as obtained M-responses during these periods. Significantly reduced H-reflex generation in fatigue compared with the pre-condition with increasing age was observed in linear regression (figure on the left) while similar amplitude M-response (figure on the right) was found for pre-fatigue and fatigue. Error bars are standard error of the mean, ${ }^{n s} p>0.05$

Fig 4 The percent change of H-reflex and comparison of the mean M-response between pre-and post-fatigue trials. No significant change of H-reflex amplitude with increasing age was observed during the recovery period for linear regression (figure on the left) and similar amplitude M-response was observed in both pre-and post-fatigue protocol (figure on the right) 